\title{
Comprendre la photosynthèse
}

\author{
Paul MATHIS \\ Service de Bioénergétique et Biologie Structurale \\ Institut de Biologie et de Technologie de Saclay, 91191 Gif-sur-Yvette Cedex \\ mathis.paul@orange.fr
}

Cette revue décrit brièvement la photosynthèse : absorption des photons, transfert d'énergie, séparation de charges, et étapes chimiques. Son intérêt pour les sciences bio-inspirées est présenté dans trois domaines : structure et fonctionnement des centres réactionnels, structure et fonctionnement du système d'oxydation de l'eau, et antennes bio-inspirées.

L es organismes vivants, dans leur variété et leur complexité, présentent de nombreuses propriétés qui sont des sources d'inspiration pour les ingénieurs. Parmi les processus biologiques qui mettent en jeu la lumière, la photosynthèse est certainement le plus complexe et le plus riche en mécanismes susceptibles d'intéresser les opticiens. Avant de discuter de son intérêt pour la bio-inspiration, il peut être utile de présenter ce qu'est la photosynthèse.

\section{La photosynthèse : généralités}

La photosynthèse est le processus par lequel les végétaux (surtout plantes et algues) et certaines bactéries, dites photosynthétiques, utilisent l'énergie de la lumière pour synthétiser leurs biomolécules. Au plan chimique, les molécules de départ sont le $\mathrm{CO}_{2}$ et l'eau ; les produits sont toutes les biomolécules, et particulièrement les molécules de réserve, hydrates de carbone et corps gras, qui permettent aux organismes de durer et de se multiplier. La photosynthèse est parfois présentée comme un mécanisme de stockage de l'énergie. C'est largement exact, mais l'aspect moléculaire, dans toute sa variété, ne doit pas être oublié.

Dans les meilleures conditions, les organismes photosynthétiques vont stocker sous forme d'énergie chimique environ $6 \%$ de l'énergie solaire qu'ils reçoivent. En moyenne, cela sera $1 \%$ ou moins, du fait de conditions défavorables (froid ou chaleur excessifs, manque d'eau, manque

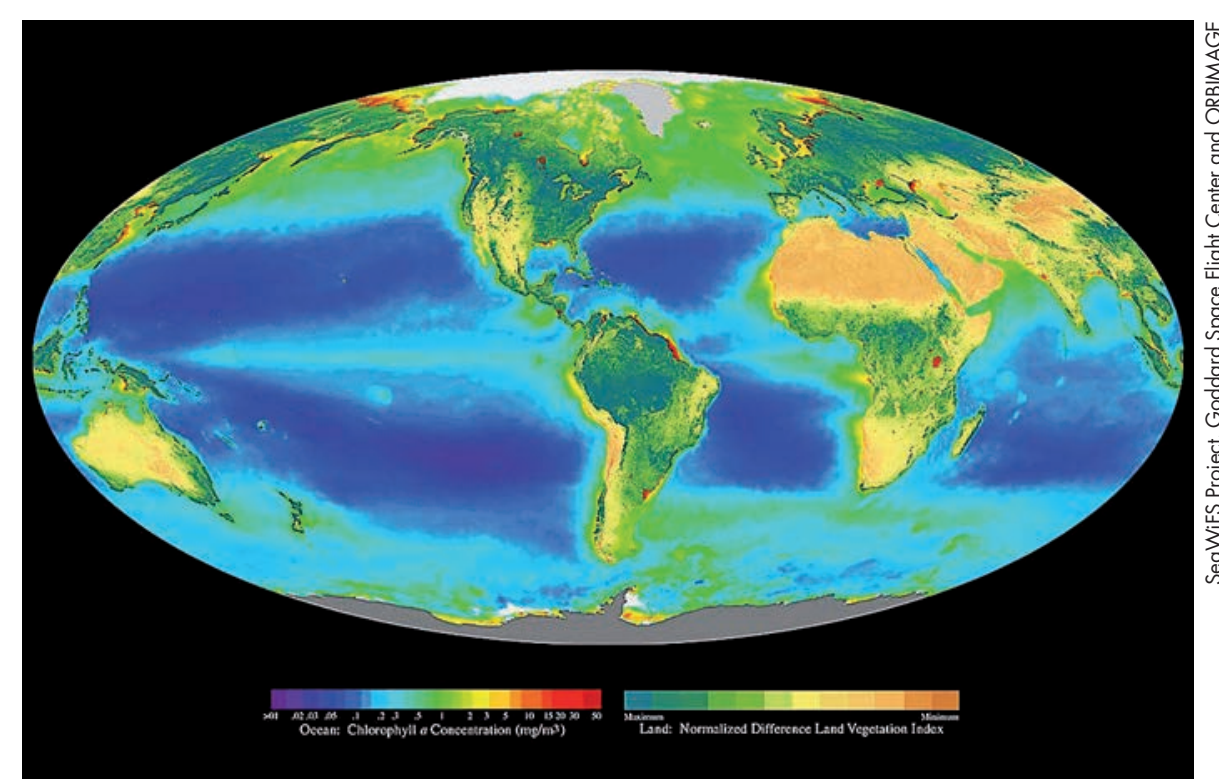

Figure 1. Vue composite montrant la distribution de l'activité photosynthétique à la surface de la Terre, le rouge foncé et le bleu-vert indiquant les zones les plus actives du phytoplancton des milieux aquatiques et de la végétation sur la terre ferme respectivement.

d'azote, etc.). À l'échelle planétaire, cela correspond pourtant à 100 milliards de tep (tonnes d'équivalent pétrole) par an, soit 8 fois la consommation d'énergie par les humains.

La photosynthèse occupe une place déterminante dans les équilibres planétaires (voir Bichat H. et Mathis P., La biomasse, énergie d'avenir? Editions Quae, 2013). En effet, elle est la source de tout le carbone organique nécessaire à la vie, en particulier à la nourriture des animaux ; elle est à l'origine de tout l'oxygène atmosphérique, celui que l'on respire, mais aussi celui qui a permis la formation de la couche d'ozone stratosphérique. C'est elle aussi qui a permis l'accumulation des combustibles fossiles qui fournissent $80 \%$ de l'énergie de nos sociétés.

\section{La photosynthèse : absorption des photons et transfert d'énergie}

Phénomène planétaire de grande échelle, la photosynthèse commence au niveau moléculaire par l'absorption de photons, dans la gamme $400-700 \mathrm{~nm}$ (étendue à $1000 \mathrm{~nm}$ chez certaines 


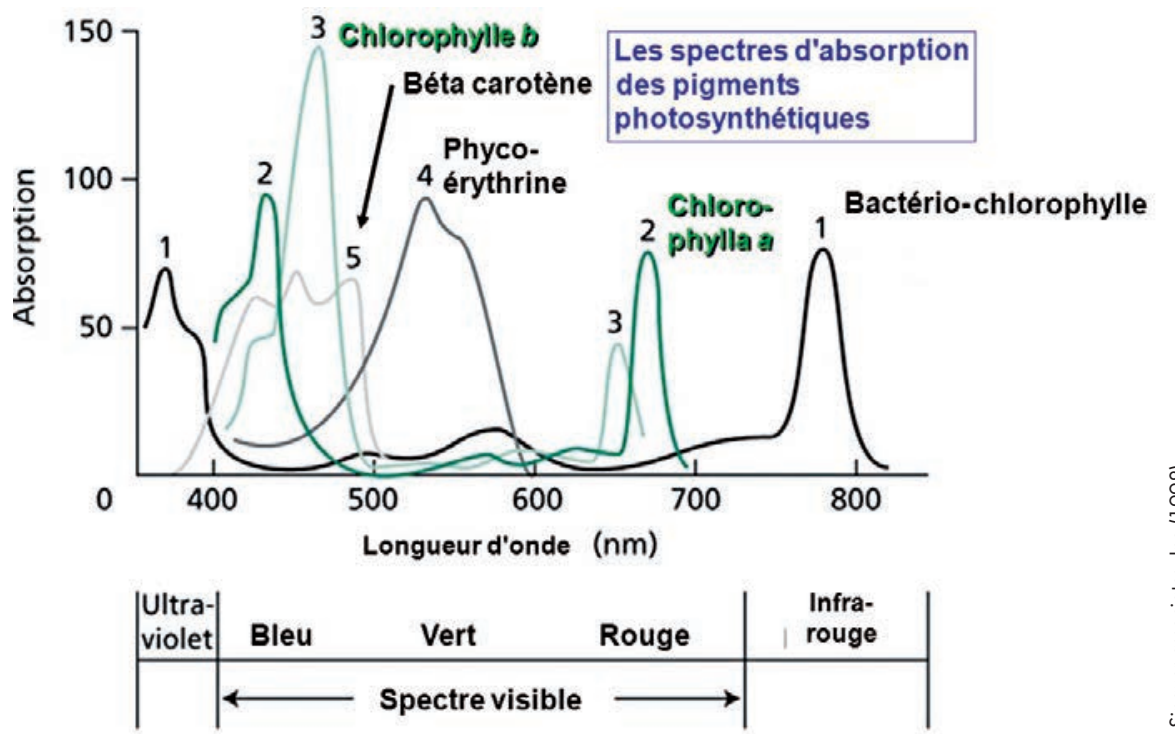

Figure 2. Spectres d'absorption des deux chlorophylles $a$ et $b$, et des autres pigments photosynthétiques.

bactéries), par des pigments variés. Ceuxci appartiennent à trois grandes classes chimiques : les chlorophylles, des tétrapyrroles cycliques à magnésium central, de couleur globalement verte, présentes dans tous les organismes photosynthétiques ; les pigments biliaires, des tétrapyrroles linéaires dont la couleur très variable est à l'origine de la multiplicité de couleurs des algues; et les caroténoïdes, des polyènes de couleur jaune orangé, dont la fonction essentielle est probablement de protéger les organismes contre les effets délétères de la lumière. La multiplicité de ces pigments permet l'absorption efficace de la lumière dans un large domaine spectral.

Une propriété essentielle de tous ces pigments est qu'ils sont organisés, c'està-dire portés en des positions très précises, à l'échelle de quelques $\AA$, par des protéines. L'absorption d'un photon par une molécule promeut celle-ci dans un état excité. L'ensemble des pigments constitue ce qu'on appelle l'« antenne». Sa fonction est d'absorber la lumière et de transférer l'énergie d'excitation jusqu'en des sites spécifiques, appelés centres réactionnels, où elle sera stockée sous forme électrochimique.

L'antenne comporte entre 20 et 1000 molécules de pigments organisées pour permettre un transfert d'énergie très rapide, en moins de 100 ps, jusqu'aux centres réactionnels. Elle est constituée de complexes protéine-pigments qui portent

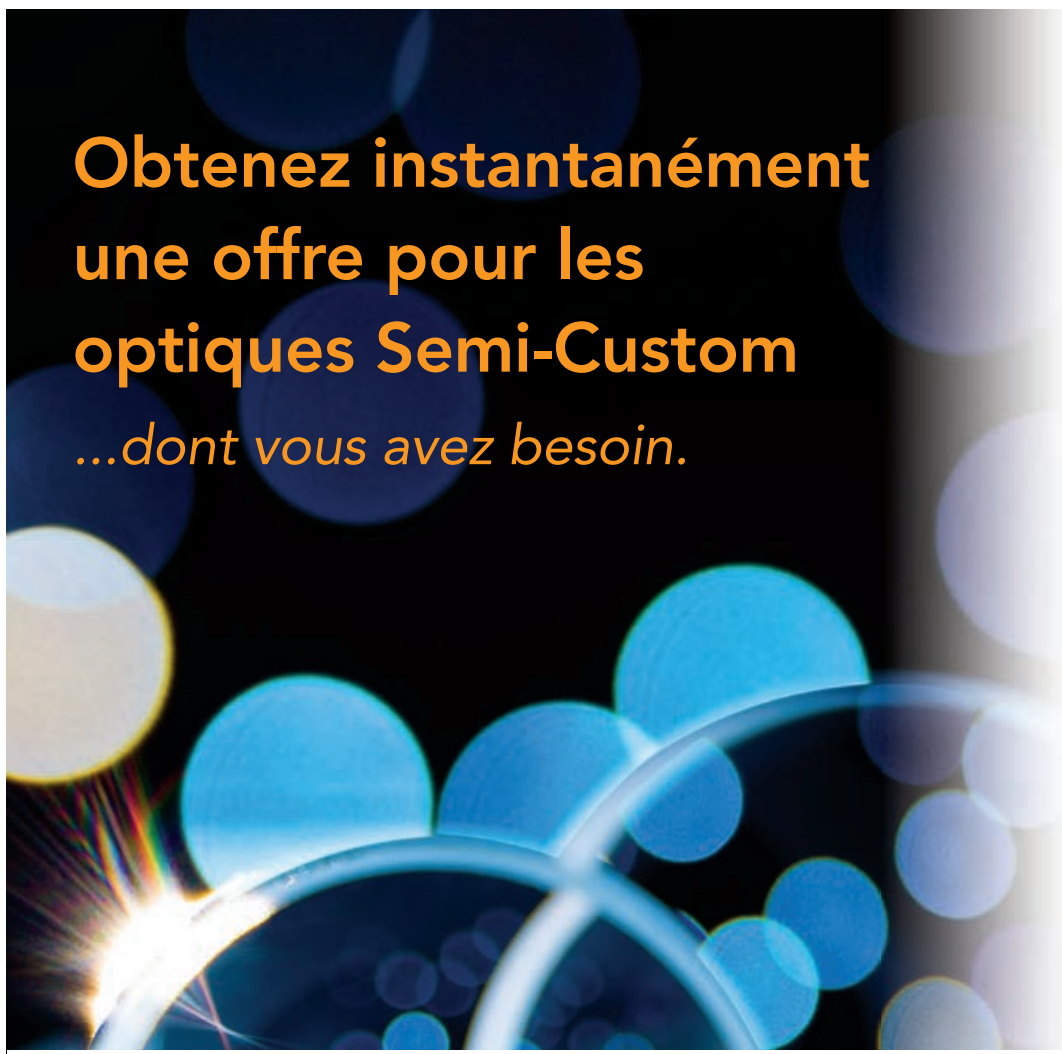

Sélectionnez et commandez en trois étapes simples et rapides votre optique semi-custom : lentille, fenêtre, miroir, séparatrice, lame retard ou filtre. Disponibles parmi une large gamme de substrats et de traitements à des prix compétitifs, tous ces produits vous sont expédiés sous 4 semaines. Précisément les optiques semi-custom dont vous avez besoin

marketplace.idexop.com +33(0) 130120680

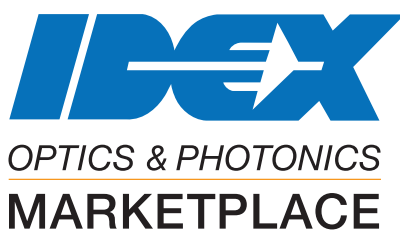

ADVANCED THIN FILMS

QV/LaserOptics 


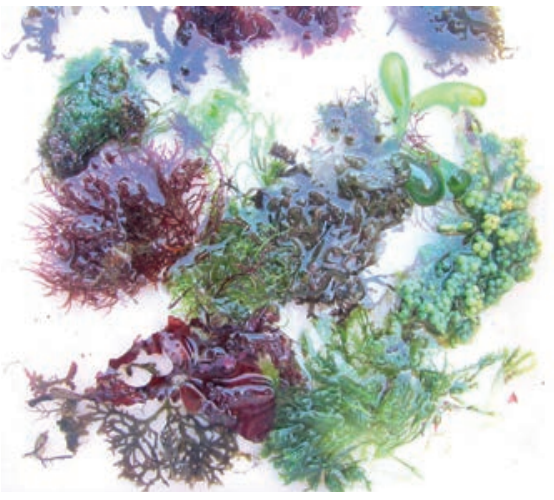

Figure 3. Variété des couleurs des algues.

les pigments mentionnés ci-dessus. Le transfert d'énergie peut être décrit, selon les cas, comme une succession de sauts d'une molécule à une voisine, ce qui aboutit, d'une façon aléatoire, à son piégeage à un centre réactionnel. Dans d'autres cas, le transfert d'énergie est de nature ondulatoire et cohérente, permettant un transfert rapide dans une antenne de grande taille. Pour assurer un bon rendement énergétique, le transfert jusqu'à un centre réactionnel doit être nettement plus rapide que la désexcitation par fluorescence ou conversion interne. De nombreux complexes protéine-pigments à fonction d'antenne ont été isolés, purifiés, cristallisés, et leur structure déterminée à haute résolution par radiocristallographie $\mathrm{X}$.

\section{La photosynthèse : séparation et transfert de charges}

Les centres réactionnels $C R$ sont les lieux où parvient l'énergie d'excitation. Celle-ci est convertie irréversiblement en énergie électrochimique par un mécanisme de séparation de charges. Les centres réactionnels sont des protéines, toujours positionnées dans des membranes et situées à proximité de complexes-antennes. Leur cœur fonctionnel est constitué d'une paire de molécules de chlorophylle (appelée bactériochlorophylle dans les bactéries). C'est cette paire, souvent appelée paire spéciale, qui sert de piège pour l'énergie. Son excitation lui permet de transférer un électron à une molécule voisine, accepteur d'électron, qui transfère à la suivante, etc. selon une cascade de transferts d'électron. La dynamique du premier est à l'échelle de la picoseconde ; les suivants sont plus lents, jusqu'à la microseconde.

Le centre réactionnel peut donc être assimilé à une photopile moléculaire. Son pôle positif est formé par la paire spéciale oxydée, dotée d'une charge positive. Son pôle négatif est constitué d'une molécule réduite, dotée d'une charge négative; selon les cas, c'est une quinone ou une protéine à fer-soufre.

\section{La photosynthèse : des étapes chimiques}

Les centres réactionnels appartiennent à plusieurs catégories qu'il serait trop compliqué de décrire ici. Leurs propriétés dépendent des réactions chimiques qu'elles vont induire dans des étapes ultérieures, en des temps supérieurs à la microseconde.

Une réaction importante est l'oxydation de molécules d'eau, avec dégagement de dioxygène et libération de protons $\mathrm{H}^{+}$. Cette réaction est déclenchée par le pôle positif de centres réactionnels dits « photosystème II » (PSII), dans les organismes dits oxygéniques, végétaux et cyanobactéries. Elle met en jeu un complexe enzymatique comportant quatre atomes de manganèse.

Dans les mêmes organismes, le pôle négatif de centres réactionnels dits «photosystème I » (PSI), constitué de protéines à fer-soufre, entraîne la réduction d'un cofacteur appelé $\mathrm{NADP}^{+}$en NADPH qui est utilisé pour la réduction du $\mathrm{CO}_{2}$ en sucres selon des réactions qui mettent en jeu un grand nombre d'enzymes.

Plusieurs réactions de transfert d'électrons entraînent le transfert de protons de part et d'autre du système membranaire où sont positionnés les centres réactionnels et les transporteurs d'électrons. Ces réactions conduisent à la création d'un potentiel membranaire dont l'énergie libre est utilisée par une protéine appelée ATPsynthase. Celle-ci produit de I'ATP, un vecteur cellulaire d'énergie qui est consommé (entre autres) pour la synthèse des sucres.

Ces sucres sont ensuite utilisés localement pour la synthèse d'autres molécules (acides aminés, corps gras, etc.) ou transportés vers les organes de réserve de la plante : fruits, tubercules, racines, tiges et troncs.

\section{Quel intérêt pour les sciences bio-inspirées?}

\section{Structure et fonctionnement des centres réactionnels}

Ces derniers ont un rendement quantique supérieur à $90 \%$ (chaque excitation entraîne pratiquement une séparation de charges) et un rendement énergétique supérieur à $50 \%$. Pourquoi une telle efficacité ? Cette question est l'objet de recherches sur la théorie des transferts

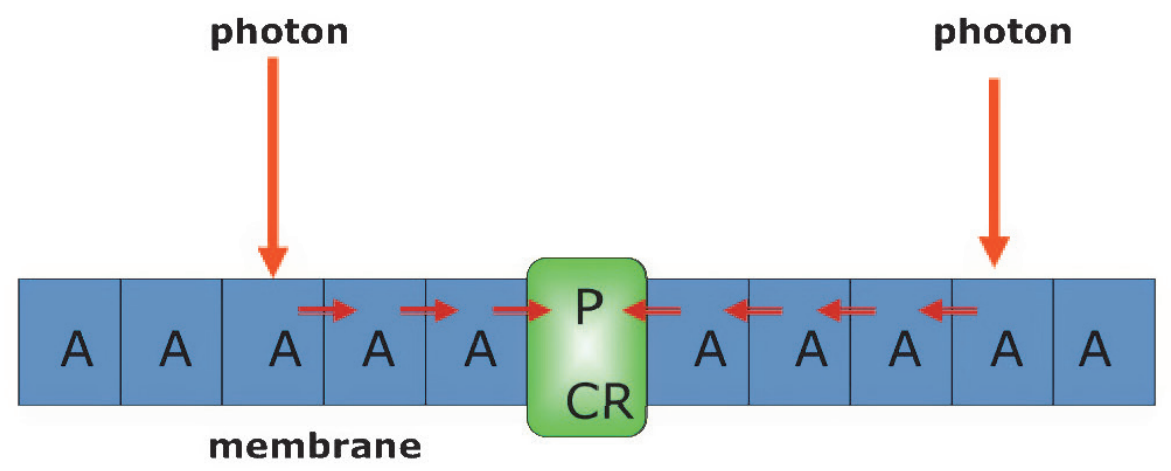
A : protéine antenne
CR : centre réactionnel
$\rightleftarrows$ Transfert d'énergie
$P$ : piège pour l'énergie

Figure 4. L'antenne : une assemblée de protéines pour la séparation et le transfert d'énergie. 


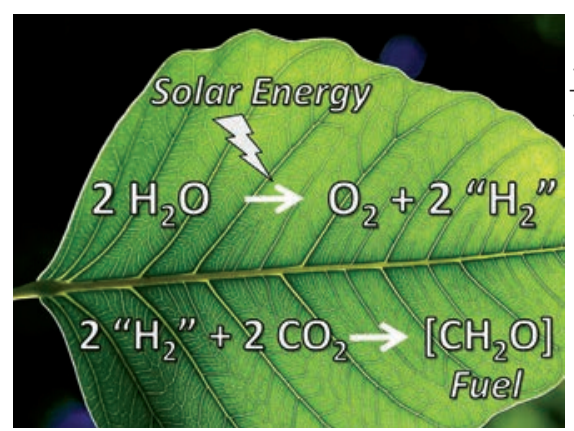

Figure 5. Artificial leaf : elle vise à décomposer l'eau en oxygène et en hydrogène, imitant le processus naturel des plantes.

d'électron, s'appuyant sur de nombreuses données expérimentales, telles que la structure précise du centre réactionnel, les propriétés électrochimiques des centres rédox, les cinétiques de transfert d'électron, les perturbations induites par mutagenèse dirigée, etc. Ce corpus de connaissances est très utile aux nombreux chimistes qui synthétisent des assemblages moléculaires qui pourraient servir pour la photosynthèse artificielle aux fins de conversion de l'énergie solaire.

\section{Structure et fonctionnement du système d'oxydation de l'eau}

Le centre réactionnel photosystème II a une masse molaire de $350 \mathrm{kDa}$, et pas moins de 20 sous-unités protéiques. Sa structure à haute résolution n'a été obtenue que très récemment. Elle montre un site catalytique d'oxydation de l'eau sous forme d'un cluster $\mathrm{Mn}_{4} \mathrm{CaO}_{5}$ associé à des ligands protéiques. Son fonctionnement est l'objet d'études approfondies. Cette question intéresse les chimistes qui souhaitent construire des systèmes biomimétiques qui oxyderaient l'eau sous l'action de la lumière. Une telle réaction produit des électrons et des protons $\mathrm{H}^{+}$, qui peuvent être les précurseurs de l'hydrogène, un combustible recherché.

\section{Des antennes bio-inspirées ?}

L'existence d'une antenne dans tous les organismes photosynthétiques est une propriété d'un grand intérêt pour tous les photochimistes. Elle permet en effet de collecter la lumière solaire, qui est par nature dispersée et donc difficile à recueillir. Cette antenne est d'autant plus intéressante que sa composition pigmentaire, sa taille et sa structure varient selon les organismes, et parfois selon les conditions d'éclairement (spectre, intensité). Elle a servi d'inspiration au chimiste suisse Michaël Graetzel pour ses cellules photoélectrochimiques. D'autres chercheurs tentent de construire des systèmes d'antennes artificielles à haute efficacité sur le modèle des structures biologiques.

Les connaissances acquises sont utilisées pour construire des systèmes intégrés entièrement synthétiques appelés feuilles artificielles (artificial leaf) intégrant des structures à fonction d'antenne, des analogues de centres réactionnels et des clusters capables d'oxyder l'eau en dioxygène. La dénomination artificial leaf est encore passablement prétentieuse, mais elle indique clairement l'objectif recherché.

Cette courte revue ne saurait couvrir tous les domaines où la photosynthèse naturelle sert de base au biomimétisme. Citons encore le cas de la production d'hydrogène. Celle-ci est effectuée par des algues et des bactéries photosynthétiques en utilisant des enzymes à métal banal, fer et nickel, là où les techniques actuelles nécessitent des métaux précieux, chers et présents en quantité limitée.

\section{Références}

Bensaid, S., Centi, G., Garrone, E., Perathoner, S. \& Saracco, G. (2012) Toward artificial leaves for solar hydrogen and fuels from carbon dioxide. ChemSusChem 5, 500-521.

Nocera, D. (2012) The artificial leaf. Acc. Chem. Res. 45, 683-776.

Sanderson, K. (2008) Chemistry: the photon trap. Nature, 452, 400-402.

Gust, D., Moore, T.A. \& Moore, A. L. (2012) Realizing artificial photosynthesis. Faraday Discuss. 155, 9-26.

Umena, Y., Kawakami, K., Shen, J.-R. \& Kamiya, N. (2011) Crystal structure of oxygen-evolving photosystem II at a resolution of $1.9 \AA$. Nature, $473,55-60$

Blankenship, R.E. Molecular mechanisms of photosynthesis. Wiley, New York, 2014, 312 p. Grätzel, M. (2001) Photoelectrochemical cells. Nature, 414, 338-344.

Faunce, T. et al. (2014) Artificial photosynthesis as a frontier technology for energy sustainability. Energy Environ. Sci. 6, 1074-1076.
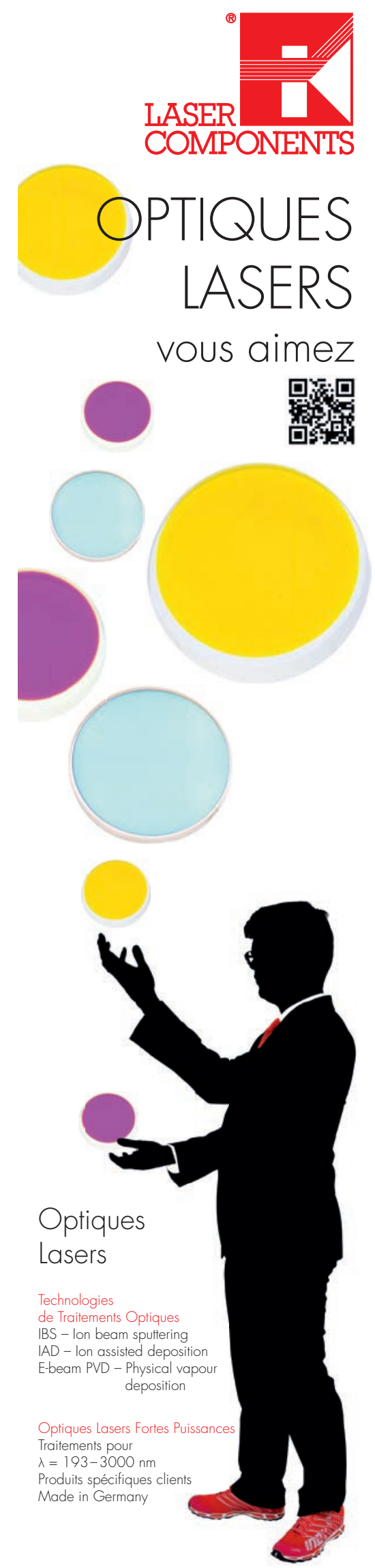

www.lasercomponents. fr 